\title{
Arterial stiffness index based on home (HASI) vs. ambulatory (AASI) blood pressure measurements
}

\author{
George S Stergiou, Anastasios Kollias, Vayia C Rarra, Efthimia G Nasothimiou and Leonidas G Roussias
}

Ambulatory arterial stiffness index (AASI) is a novel index derived from the linear relationship between 24-h ambulatory systolic and diastolic blood pressure (BP) measurements. This study investigated whether 'home arterial stiffness index' (HASI) based on self-home BP measurements is similar to AASI. A total of 483 hypertensive subjects underwent 24-h ambulatory and 6-day home BP monitoring. AASI and HASI were defined as one minus the respective regression slope of diastolic on systolic BP. Mean HASI $(0.66 \pm 0.17)$ was higher than $24-h(0.33 \pm 0.15)$ daytime $(0.50 \pm 0.18)$ and nighttime AASI $(0.37 \pm 0.19$, $P<0.001$ for all comparisons vs. HASI) and was weakly correlated with 24 -h $(r=0.14, P<0.01)$ daytime $(r=0.14, P<0.01)$ and nighttime AASI $(r=0.09, P=0.05)$. Compared to 24-h AASI, HASI was less closely associated with age $(r=0.46$ and 0.10 respectively, $P<0.001$ for difference), systolic home $\mathrm{BP}(r=0.30$ and $0.09, P<0.001)$ and pulse pressure $(r=0.52$ and 0.20 , $P<0.001)$, as well as with 24-h ambulatory systolic BP $(r=0.21$ and $0.05, P<0.05)$ and pulse pressure $(r=0.56$ and 0.25 , $P<0.001$ ). No satisfactory agreement was observed between HASI and 24-h (agreement $63 \%, \kappa 0.02$ ) daytime (agreement $65 \%, \kappa 0.1$ ) or nighttime AASI (agreement $63 \%, \kappa 0.03$ ) in detecting subjects at the top quartile of the respective distributions. HASI appears to be similar but also has important differences from AASI and is less closely associated with markers of arterial stiffness. These data do not support the view that home BP measurements can replace ambulatory monitoring in the assessment of the arterial stiffness index.

Hypertension Research (2010) 33, 731-736; doi:10.1038/hr.2010.62; published online 30 April 2010

Keywords: ambulatory blood pressure monitoring; arterial stiffness index; home blood pressure

\section{INTRODUCTION}

Ambulatory arterial stiffness index (AASI) derived from the linear relationship between 24-h ambulatory systolic and diastolic blood pressure (BP) measurements has been recently proposed as a measure of arterial stiffness. ${ }^{1}$ AASI has been found to be highly correlated with classic measures of arterial stiffness, including aortic pulse wave velocity (PWV), central and peripheral pulse pressure and the systolic augmentation index. ${ }^{2,3}$ Most importantly, recent studies showed an association of AASI with signs of target organ damage, as well as with non-fatal and fatal strokes and cardiovascular mortality, over and beyond pulse pressure and other risk factors. ${ }^{3-6}$ Taking into account the aforementioned data and that the classic methods of arterial stiffness evaluation require special expensive equipment and trained observers, AASI emerges as an especially applicable measure in clinical practice.

As it is the case with ambulatory BP (ABP) monitoring, selfmonitoring of BP by patients at home (HBP) also provides multiple BP measurements away from the clinic setting in the usual environment of each individual. Moreover, HBP has been shown to be a reliable alternative to $\mathrm{ABP}$ for the diagnosis of white coat and masked hypertension, ${ }^{7}$ at least as closely associated with target organ damage as $\mathrm{ABP}^{8-10}$ and a strong predictor of cardiovascular events risk. ${ }^{11}$
However, it is not known whether arterial stiffness index calculated using home BP measurements is a reliable alternative to AASI. As HBP monitoring is less costly, more widely available and better accepted by patients than $\mathrm{ABP}$ monitoring, the application of 'home arterial stiffness index' (HASI), if proved reliable, would have considerable potential for wide use in clinical practice. ${ }^{12}$

This study was designed to test the hypothesis that HASI provides similar information and, therefore, is a useful alternative to AASI for clinical practice.

\section{METHODS}

\section{Participants}

Study subjects were untreated or treated adults referred to an outpatient hypertension clinic for elevated BP. All participants had their BP assessed using a standard protocol for clinic $\mathrm{BP}$ (CBP), ABP and HBP monitoring in the context of prospective clinical trials conducted from 1995 to 2008. Antihypertensive treatment should remain unchanged 4 weeks before and throughout the study. Each subject could enter the database for analysis in untreated and treated state, but once per treatment status and with the first set of BP measurements if more than one were available. The study protocol for retrospective analysis of patients' data has been approved by the hospital scientific committee. 


\section{BP measurements}

$\mathrm{CBP}, \mathrm{ABP}$ and $\mathrm{HBP}$ measurements were performed within 4 weeks. $\mathrm{CBP}$ measurements were taken during one study visit, after at least one pre-study visit. Measurements were taken by physicians who fulfilled the British Hypertension Society Protocol criteria for observers' agreement in BP measurement. ${ }^{13}$ Triplicate CBP measurements were performed at each visit after $5 \mathrm{~min}$ sitting rest and with at least 1 min between measurements using standard mercury sphygmomanometers (inflatable bladder size $12 \mathrm{~cm} \times 24 \mathrm{~cm}$ or $15 \mathrm{~cm} \times 35 \mathrm{~cm}$ according to the individuals' arm circumference).

HBP was monitored on six routine workdays within 2 weeks using validated fully automated electronic devices Omron HEM-705CP or OMRON IC (Omron Healthcare GmbH, Hamburg, Germany; bladder size $12 \mathrm{~cm} \times 23 \mathrm{~cm}$ or $14 \mathrm{~cm} \times 28 \mathrm{~cm}$ as appropriate). Participants were trained in the conditions of HBP measurement and the use of the devices and were instructed to perform duplicate morning (6-9 AM, before drug intake if treated) and evening (6-9 PM) measurements after $5 \mathrm{~min}$ sitting rest and with $1 \mathrm{~min}$ between recordings. A form was supplied to the participants to report their HBP values. These data were verified by printing all measurements through the devices' memory (HEM-705CP) or the PC link function (IC).

ABP was monitored on a routine workday, before or after the HBP monitoring period by using validated oscillometric devices SpaceLabs 90207 or 90217 (SpaceLabs, Redmond, WA, USA; bladder size $12 \mathrm{~cm} \times 23 \mathrm{~cm}$ or $14 \mathrm{~cm} \times 30 \mathrm{~cm}$ as appropriate; measurements at 15-20-min intervals for $24 \mathrm{~h}$ ). Subjects were instructed to remain still with their arm extended and relaxed during each measurement. Before each ABP or HBP monitoring session the accuracy of each device was tested against a standard mercury sphygmomanometer by manual activation to ensure that there was no consistent difference of $>10 \mathrm{~mm} \mathrm{Hg}$ in the measured BP (three successive readings; $\mathrm{Y}$ connector). Day and night periods were defined according to the individual patients' diaries. A non-dipping pattern was defined as a $<10 \%$ fall in systolic and/or diastolic BP during nighttime sleep as compared to awake measurements.

\section{AASI and HASI estimation}

The regression slope of diastolic on systolic BP was computed for each individual on the basis of ABP (AASI) and also of HBP readings (HASI). AASI as well as HASI were defined as one minus the respective regression slope. AASI was also computed from awake or asleep BP recordings only (daytime and nighttime AASI, respectively). Symmetrical AASI and HASI were also calculated using the respective formulas: $1-(1-\mathrm{AASI}) / r_{1}$ and $1-(1-\mathrm{HASI}) / r_{2}$, with $r_{1}$ and $r_{2}$ being the correlation coefficients between systolic and diastolic $\mathrm{ABP}$ and $\mathrm{HBP}$ values, respectively. ${ }^{14-16}$

\section{Statistical analysis}

Subjects with less than 11 valid awake or 7 asleep ABP readings or less than 20 valid HBP readings were excluded. The ABP data and additional recorded information from the report files generated by the ABP monitor device were batch imported and organized in a relational database (Microsoft Access 2000; Microsoft, Redmond, WA, USA) using a Visual Basic program. This program, designed by LGR for statistical analysis of ABP-derived data, reads the ASCII text files generated by the ABP monitor and performs multiple data procedures and analyses. Systolic ambulatory and home BP readings $<60$ or $>250 \mathrm{~mm} \mathrm{Hg}$ or diastolic $<30$ or $>150 \mathrm{~mm} \mathrm{Hg}$ were excluded, as were ambulatory measurements flagged by the software of the monitors as being technically erroneous. Ambulatory readings taken $<20 \mathrm{~min}$ after the monitor had been attached to subjects were also excluded because these had been taken in the clinic.

Pearson's correlations and multiple regression analyses were performed to investigate the association of AASI and HASI with anthropometric variables. Paired $t$-tests were used to compare AASI and HASI values of the same subjects. Bonferroni's correction for multiple comparisons was applied where appropriate. The agreement between AASI and HASI in detecting subjects at the top quartile of their respective distributions was estimated using the $\kappa$ statistic. Coefficient of variation was calculated as the standard deviation divided by the mean value. Standard deviation values were compared using the $F$-test. Results are expressed as mean values with s.d. A probability value $P<0.05$ was considered statistically significant. Statistical analysis was performed using the
Statistical Package for Social Sciences software (SPSS release 13.0; SPSS, Chicago, IL, USA).

\section{RESULTS}

\section{Participants characteristics}

A total of 710 cases were reviewed for eligibility and 227 were excluded. One hundred and forty cases were excluded because of inadequate HBP and/or ABP readings, 34 because evaluation had been performed more than once in the same treatment status (untreated/ treated), 39 because of unknown sleeping hours, 5 because of treatment changes during the BP data collection, 5 because of acute disease during the study and 4 because of other reasons. The final analysis included 483 cases. The main characteristics of the study population are shown in Table 1 . The average number of $24-\mathrm{h} \mathrm{ABP}$ readings analyzed was $67.1 \pm 5.2(42 \pm 6.9$ for the daytime and $25 \pm 5.7$ for the nighttime period). The average number of HBP readings analyzed $(23.3 \pm 1.2)$ was lower than that of the daytime or the nighttime ABP monitoring period ( $P<0.001$ for both comparisons). The ratio of the number of daytime to nighttime ABP readings was $1.8 \pm 0.8$. Thirtyfour percent of the subjects exhibited a non-dipping pattern concerning systolic and/or diastolic ABP.

\section{Variation of HBP vs. ABP measurements}

The coefficient of variation was lower for diastolic HBP values compared with ABP (0.11 vs. 0.124), and higher for systolic HBP measurements compared with ABP (0.106 vs. 0.098). The ratio of the coefficient of variation of diastolic to that of systolic BP measurements was lower for home (1.038) compared with ABP (1.265). Furthermore, the standard deviation of diastolic HBP tended to be lower than of $\mathrm{ABP}$ measurements $(9.4$ vs. 10.2, $P=0.06)$ and was higher for systolic HBP compared to ABP measurements (14.5 vs. 12.8, $P=0.005)$.

Table 1 Participants' characteristics, blood pressure levels and arterial stiffness index values

\section{Characteristic}$$
n
$$

Age (years)

Males (\%)

$52.2 \pm 12.3$

Height $(\mathrm{cm})$ 289 (59.8)

Weight (kg)

$168.6 \pm 9.5$

$79.9 \pm 14.3$

Body mass index $\left(\mathrm{kg} \mathrm{m}^{-2}\right)$

$28.1 \pm 4.0$

Treated for hypertension (\%)

179 (37.1)

Office systolic BP $(\mathrm{mm} \mathrm{Hg})$

$140.1 \pm 15.3$

Office diastolic BP $(\mathrm{mm} \mathrm{Hg})$

$89.7 \pm 10.7$

Office pulse pressure $(\mathrm{mm} \mathrm{Hg})$

$50.4 \pm 12.3$

24-h systolic BP ( $\mathrm{mm} \mathrm{Hg}$ )

$131.1 \pm 12.8$

24-h diastolic BP $(\mathrm{mm} \mathrm{Hg})$

$82.0 \pm 10.2$

24-h pulse pressure $(\mathrm{mm} \mathrm{Hg}$ )

$49.1 \pm 8.3$

24-h pulse rate (beats per $\mathrm{min}$ )

$72.3 \pm 9.1$

Home systolic BP $(\mathrm{mm} \mathrm{Hg})$

$136.8 \pm 14.5$

Home diastolic BP $(\mathrm{mm} \mathrm{Hg})$

$85.7 \pm 9.4$

Home pulse pressure $(\mathrm{mm} \mathrm{Hg})$

$51.1 \pm 11.4$

AASI

$0.33 \pm 0.15$

Daytime AASI

$0.50 \pm 0.18$

Nighttime AASI

$0.37 \pm 0.19$

HASI

$0.66 \pm 0.17$

Abbreviations: AASI, ambulatory arterial stiffness index; BP, blood pressure; HASI, home arterial stiffness index. 


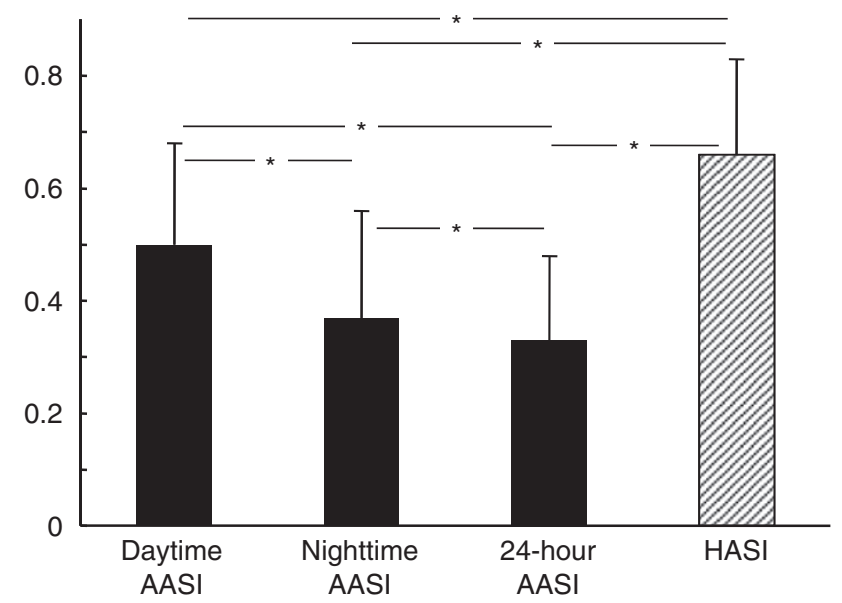

Figure 1 Comparison of home arterial stiffness index (HASI) with 24-h, daytime and nighttime arterial stiffness indexes (AASI) (mean \pm s.d.). ${ }^{*} P<0.001$

\section{HASI vs. AASI}

Mean HASI was higher than 24-h, daytime and nighttime AASI (Figure 1). Daytime AASI was higher than nighttime AASI (Figure 1) and 24-h AASI was lower than daytime and nighttime AASI (Figure 1). Women had higher AASI values than men (0.36 \pm 0.16 and $0.32 \pm 0.14$, respectively, $P=0.001)$, whereas no such difference in HASI was found $(0.66 \pm 0.16$ and $0.65 \pm 0.17$, respectively, $P=\mathrm{NS})$. Treated subjects had higher AASI values compared with untreated ones $(0.36 \pm 0.15$ and $0.32 \pm 0.14$, respectively, $P<0.001)$, with no difference in HASI $(0.66 \pm 0.16$ and $0.64 \pm 0.17$, respectively, $P=\mathrm{NS})$. Dippers had lower AASI values than non-dippers $(0.3 \pm 0.13$ and $0.4 \pm 0.15$, respectively, $P<0.001)$ but similar HASI levels $(0.66 \pm 0.17$ and $0.65 \pm 0.16$ respectively, $P=\mathrm{NS}$ ).

\section{Correlations and multivariate analyses}

Correlations of HASI and AASI values with several demographic and $\mathrm{BP}$ variables are presented in Table 2. AASI was significantly correlated with age, clinic, ambulatory and home systolic BP and pulse pressure and the number of antihypertensive drugs, and inversely with weight, height, diastolic CBP, ABP and HBP and 24-h pulse rate (Table 2). HASI was weakly correlated with AASI values (Figure 2) and gave similar correlations and to the same direction as AASI, particularly with pulse pressure and diastolic BP values (clinic, ambulatory and home), but the strength of these associations was consistently weaker than those of AASI (Table 2). Twenty-four hour pulse pressure was strongly correlated with daytime and nighttime AASI $(r=0.39$ and 0.31 , respectively) and less so with HASI $(r=0.25, P<0.001$ and $P<0.01$, respectively, for differences in $r$ values obtained by HASI vs. daytime or nighttime AASI).

In multiple regression analysis with AASI as dependent variable and age, sex, height, weight, 24-h pulse pressure, pulse rate, day to night number of BP recordings ratio, number of antihypertensive drugs and non-dipping pattern as independent variables, only age $(\beta=0.003 \pm 0$, $P<0.001)$, 24-h pulse pressure $(\beta=0.008 \pm 0.001, P<0.001)$ and nondipping $(\beta=0.059 \pm 0.011, P<0.001)$ were associated with increased AASI $\left(R^{2}=0.45\right)$. In a similar analysis of HASI (dependent variable) with the same independent variables (except for day to night number of readings ratio), only 24 -h pulse pressure $(\beta=0.005 \pm 0.001$, $P<0.001)$ was associated with increased HASI $\left(R^{2}=0.08\right)$.
Table 2 Bivariate correlation coefficients of several demographic and blood pressure variables with home (HASI) and ambulatory arterial stiffness index (AASI)

\begin{tabular}{|c|c|c|c|}
\hline & $A A S I$ & HASI & $P(A A S I$ vs. HASI) \\
\hline AASI & - & $0.14^{* *}$ & \\
\hline Daytime AASI & $0.68^{* * *}$ & $0.14^{* *}$ & $* * *$ \\
\hline Nighttime AASI & $0.44^{* * *}$ & 0.09 & $* * *$ \\
\hline Age & $0.46^{* * *}$ & $0.10^{*}$ & $* * *$ \\
\hline Height & $-0.21^{* * *}$ & 0.00 & $* *$ \\
\hline Weight & $-0.12^{* *}$ & 0.08 & ** \\
\hline Body mass index & 0.02 & $0.12^{*}$ & NS \\
\hline Number of drugs ${ }^{a}$ & $0.25^{* * *}$ & 0.01 & $* * *$ \\
\hline Systolic clinic BP & $0.18^{* * *}$ & -0.01 & $* *$ \\
\hline Systolic 24-h BP & $0.21^{* * *}$ & 0.05 & * \\
\hline Systolic home BP & $0.30^{* * *}$ & $0.09^{*}$ & $* * *$ \\
\hline Diastolic clinic BP & $-0.28^{* * *}$ & $-0.17^{\star \star *}$ & NS \\
\hline Diastolic 24-h BP & $-0.20^{* * *}$ & $-0.14^{* *}$ & NS \\
\hline Diastolic home BP & $-0.16^{* \star *}$ & $-0.1^{*}$ & NS \\
\hline Pulse pressure clinic & $0.47^{* * *}$ & $0.13^{* *}$ & $* * *$ \\
\hline Pulse pressure 24-h & $0.56^{* * *}$ & $0.25^{* * *}$ & *** \\
\hline Pulse pressure home & $0.52^{* * *}$ & $0.20^{* * *}$ & $* * *$ \\
\hline 24-h pulse rate & $-0.23^{* * *}$ & -0.03 & ** \\
\hline
\end{tabular}

Abbreviation: BP, blood pressure; NS, not significant.

aFor hypertension.

${ }^{*} P<0.05,{ }^{* *} P \leqslant 0.01,{ }^{* * *} P \leqslant 0.001$.

\section{Assessment of systolic-diastolic correlation coefficients and} symmetrical AASI and HASI values

Mean values of correlation coefficients between systolic and diastolic $\mathrm{ABP}$ and $\mathrm{HBP}$ values were $0.80 \pm 0.10$ and $0.57 \pm 0.23$, respectively. The distribution of systolic-diastolic BP correlation coefficients in relation to the AASI and HASI values is presented in Figure 3. For systolic-diastolic HBP coefficient values $>0.5(n=303)$, mean HASI was again higher than AASI $(0.57 \pm 0.13$ vs. $0.33 \pm 0.14, P<0.001)$. In addition, in this subgroup HASI was more weakly correlated than AASI with age ( $r=0.25$ and 0.48 , respectively, $P=0.001$ for difference) and 24 -h pulse pressure $(r=0.22$ and 0.53 , respectively, $P<0.001$ for difference). When symmetrical values were examined in the total sample, mean HASI was still higher than AASI (0.38 \pm 0.2 vs. $0.17 \pm 0.15, P<0.001)$, whereas their correlation was not higher compared to that of non-symmetrical values $(r=0.19$ and 0.14 , respectively, $P=0.43$ for difference). Symmetrical AASI values were not correlated with systolic-diastolic ABP correlation coefficients; however, symmetrical HASI values were positively correlated with HBP correlation coefficients (Figure 4). Moreover, symmetrical AASI compared to symmetrical HASI was more strongly correlated with age ( $r=0.41$ and 0.26 , respectively, $P=0.008$ for difference) and 24 -h pulse pressure ( $r=0.46$ and 0.10 , respectively, $P<0.001$ for difference).

\section{Diagnostic agreement studies}

Of the 483 participants, 34 were at the top quartile of both the HASI and the AASI distribution, 88 were at the top quartile of the HASI but not the AASI distribution and 92 the reverse. No satisfactory agreement was observed between HASI and 24-h AASI (agreement 63\%, $\kappa 0.02$ ), daytime (agreement $65 \%, \kappa 0.1$ ) or nighttime AASI (agreement $63 \%, \kappa 0.03$ ) in detecting subjects in the top quartile of the respective distributions.

\section{DISCUSSION}

AASI has recently attracted attention as a novel index of arterial stiffness. $^{2-6}$ The main practical advantage of AASI is the simplicity in 

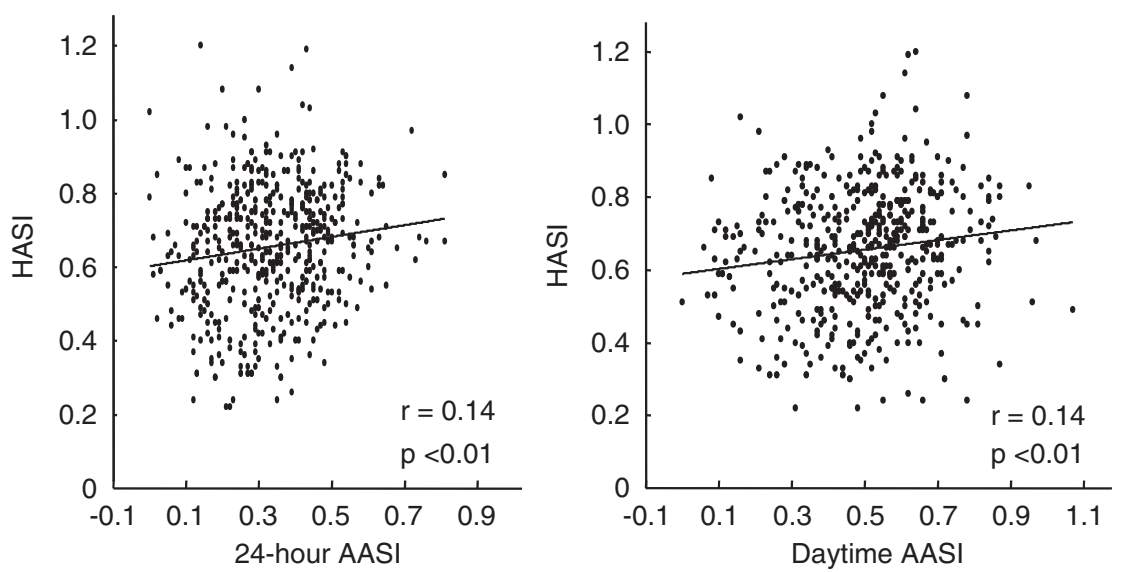

Figure 2 Correlation between home arterial stiffness index (HASI) and 24-h or daytime arterial stiffness index (AASI).
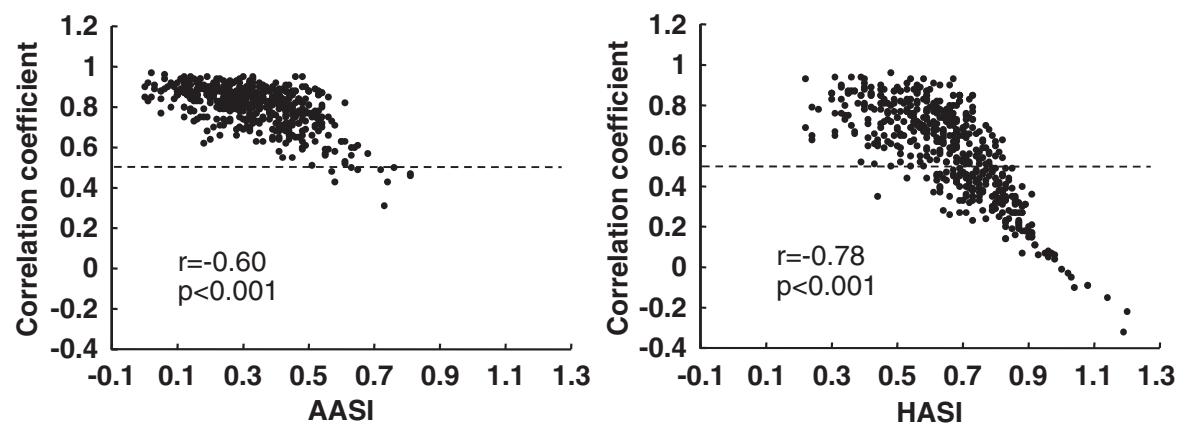

Figure 3 Association of correlation coefficients of systolic-diastolic ambulatory or home blood pressure measurements with 24-h ambulatory arterial stiffness index (AASI) or home arterial stiffness index (HASI).
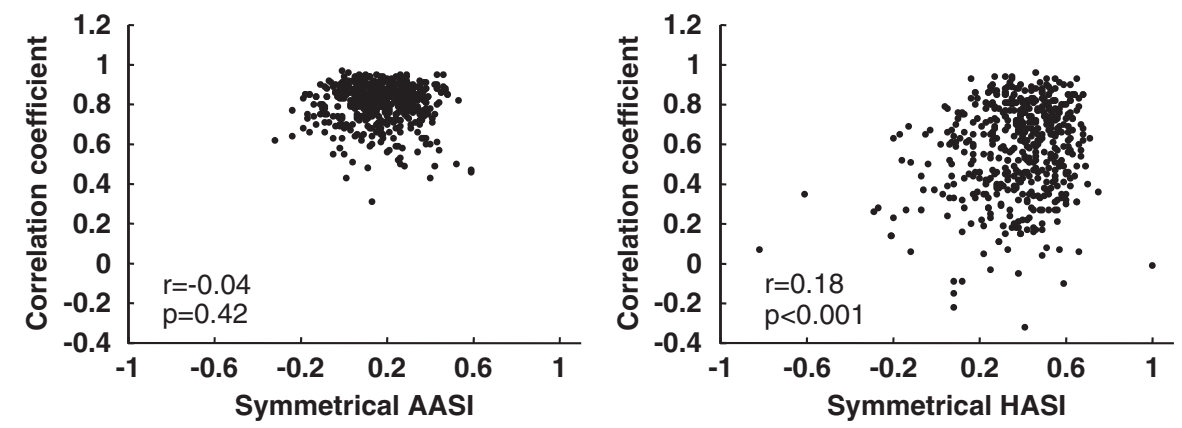

Figure 4 Association of correlation coefficients of systolic-diastolic ambulatory or home blood pressure measurements with symmetrical 24-h ambulatory arterial stiffness index (AASI) or home arterial stiffness index (HASI).

its derivation, as it does not require special equipment or qualified observers. Owing to the wide use of home BP monitoring, arterial stiffness index derived from such measurements (HASI) has greater potential for widespread application in clinical practice. One previous study by Qureshi et al. ${ }^{17}$ in 59 subjects investigated the usefulness of HASI on the basis of eight or more HBP readings and reported significant correlations with age and pulse pressure and marginally with PWV. In an attempt to investigate whether HBP can replace ABP monitoring for the estimation of the arterial stiffness index, this study analyzed HASI values in a much larger sample $(n=483)$ with an adequate number of home BP measurements $(n=23)$ and provided a direct comparison vs. AASI, yet PWV measurements were not available.
The main findings of this study are that HASI values are (i) higher than AASI, (ii) weakly correlated with AASI and (iii) associated with age, BP and pulse pressure, as it is the case for AASI values but with significantly weaker correlations. Other factors known to be related to AASI, such as gender, treatment status and dipping pattern, did not appear to affect HASI. The higher levels of HASI compared to AASI values, the weak correlation between them and their poor agreement in detecting subjects at the top quartile of the arterial stiffness index distribution imply important differences between these two BP-derived measures.

The study data in a relatively large sample of untreated and treated hypertensives confirmed previous reports showing strong correlations of AASI values with age, systolic BP and pulse pressure and inversely 

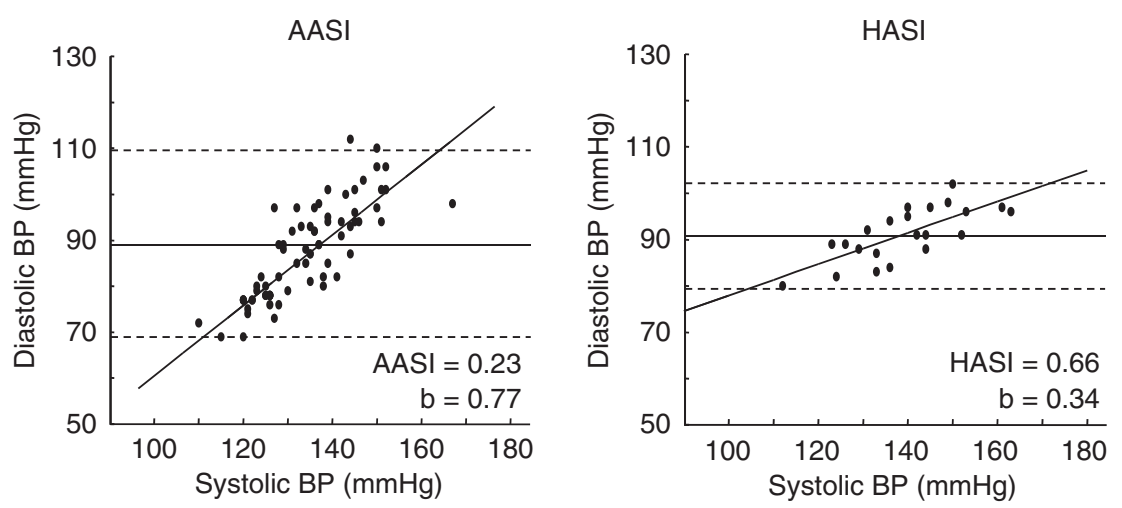

Figure 5 Distribution of diastolic on systolic blood pressure (BP) values used to calculate $24 \mathrm{~h}$ ambulatory (AASI) and home arterial stiffness index (HASI) from the same subject (horizontal lines represent mean diastolic blood pressure \pm 2 s.d.).

with diastolic BP, height and 24-h pulse rate. ${ }^{18,19}$ The strong correlations of AASI with age, which is a major predictor of arterial stiffness, and with pulse pressure (irrespective of the measurement method, clinic, ambulatory or home) that is also an established marker of arterial stiffness, are particularly re-assuring for the clinical relevance of AASI. On the other hand, HASI showed similar characteristics regarding its relationship with age and pulse pressure measurements, but with significantly weaker associations (Table 2), suggesting that it might be inferior to AASI as a marker of arterial stiffness. This drawback of the HASI is also suggested by the low ability of a multiple regression model to explain its variance as compared to AASI.

In this study, AASI and HASI values have been obtained in the same subjects, within 4 weeks time, without change in treatment and using validated oscillometric BP monitors. Thus, the same vascular and hemodynamic characteristics were assessed by these two indexes. However, there are several differences between HBP and ABP monitoring that might be responsible for the observed differences between HASI and AASI. First, HASI is exclusively based on BP measurements taken during daytime, whereas AASI is based on daytime and also sleeptime measurements. Indeed, in accordance with previous reports, daytime AASI calculated from awake ambulatory measurements was higher compared to 24-h AASI. ${ }^{20}$ However, HASI in this study remained significantly higher than daytime AASI (Figure 1). Second, HASI is based on BP measurements taken under strictly standardized conditions of posture and activity (in the sitting posture and after a few minutes rest), whereas AASI is based on measurements taken in ambulatory conditions while subjects undertake their usual daily activities. However, nighttime AASI that is also based on standardized (in bed) measurements was also significantly lower than HASI, suggesting that other factors are mainly responsible for the difference between HASI and AASI values. More importantly, nighttime AASI was more closely associated with $24 \mathrm{~h}$ pulse pressure than HASI. Third, the calculation of HASI is based on a smaller sample of BP readings compared to AASI (average of 23 and 67 readings, respectively). However, as mentioned above, nighttime AASI that is based on a similar number of BP readings ( 25 on average) was more closely related with pulse pressure than HASI.

Schillaci et al. ${ }^{21}$ suggested that a narrow range of diastolic BP values throughout the 24-h period, as it is the case in non-dippers, results into higher AASI values. ${ }^{21,22}$ This is because a narrow range of diastolic BP values flattens the regression line of diastolic on systolic BP. In the same line, for a given range of diastolic BP values, the wider the range of systolic BP values the more flat the regression line and, thereby, the higher the AASI value. Thus, the differences in the relationship of diastolic with systolic BP variation (demonstrated by the ratio of their coefficients of variation) between home and ambulatory measurements probably explain the higher HASI values compared to AASI. Moreover, the fact that the ratio of the coefficient of variation of diastolic to systolic BP was above 1 with both home and ambulatory measurements, suggests that the impact of the diastolic BP variation in increasing the arterial stiffness value was greater than of systolic. Figure 5 shows a representative case that demonstrates the differences in the distribution of diastolic on systolic $\mathrm{BP}$ values using ambulatory or home BP measurements in the same subject. It is clear in this case that diastolic BP had a narrower variation at home compared with ambulatory monitoring, which gave a more flat regression line and higher arterial stiffness index. It should be noted, however, that the diastolic BP decline during nighttime sleep probably accounts, at least in part, for the larger diastolic BP variation during ABP compared with HBP monitoring. These data also confirm previous reports that daytime AASI is higher than 24-h AASI. ${ }^{20}$

It might therefore be argued that because HASI is exclusively derived from morning and evening BP measurements, each time in duplicate but within a short time interval, this index provides only limited information for the dynamic relationship of systolic with diastolic BP. On the other hand, AASI is based on measurements obtained in a wide range of physical activity levels (during a variety of daily activities, at work, at home and during sleep), as well as measurements in the transition periods between wakefulness and sleep (nocturnal BP dip and morning surge). Therefore, AASI appears to be more appropriate for the investigation of the regression slope of diastolic on systolic BP.

The systolic-diastolic BP correlation has an important role in the determination of both AASI and HASI values and, indeed, a strong negative association was observed between AASI or HASI and the respective systolic-diastolic BP correlation coefficients (Figure 3). However, even when the analysis was restricted in the subgroup with systolic-diastolic HBP correlation coefficient values above 0.5, the results concerning the comparison of AASI and HASI or their relationship with age and pulse pressure did not change. Interestingly, when symmetrical AASI and HASI values were examined to correct for their dependence on systolic-diastolic correlation, the results were similar to those of non-symmetrical AASI and HASI values. It should be noted, however, that although symmetrical AASI was independent of the systolic-diastolic BP association, a weak positive correlation was revealed between symmetrical HASI values and systolic-diastolic BP correlation coefficients. 
The main limitation of this study is that HASI was investigated as a potential marker of stiffness by assessing its relationship with other surrogate measures of arterial stiffness (AASI and pulse pressure) rather than with direct measures of arterial stiffness (for example, PWV). However, several studies have shown that AASI and pulse pressure are reliable indirect markers of arterial stiffness and are independently associated with cardiovascular morbidity and mortality. ${ }^{3-6,23-25}$ On the other hand, there are studies that questioned the clinical relevance of AASI in predicting arterial stiffness and target organ damage. Schillaci et al. ${ }^{21}$ reported significant relationship of AASI with left ventricular mass index and PWV in untreated hypertensives, yet both associations were diminished after adjustment for other variables. Likewise, Jerrard-Dunne et al. ${ }^{26}$ found significant relationships of AASI with PWV and augmentation index in hypertensive subjects, which were lost after adjustment for age. Finally, Muxfeldt et al. ${ }^{27}$ showed that in patients with resistant hypertension, 24-h pulse pressure compared to AASI was more closely associated with arterial stiffness (PWV). Therefore, the findings of this study that HASI is not well correlated with AASI do not exclude its likelihood to correlate with arterial stiffness evaluated with a standard method such as PWV.

In conclusion, this study provides conclusive evidence on arterial stiffness index based on home BP measurements (HASI), compared to AASI obtained in the same subjects. HASI appears to have similarities with AASI, but also important differences. It has higher values and is less closely associated with established markers of arterial stiffness, such as age and pulse pressure. In multivariate analysis, HASI was determined only by pulse pressure and to a smaller degree. Poor agreement was observed between HASI and AASI in detecting subjects at the top quartile of their distributions. These data do not support the view that home measurements are interchangeable with ambulatory measurements in the calculation of the arterial stiffness index. Until the association of HASI with established indexes of arterial stiffness and cardiovascular events risk is investigated, HASI should not be regarded as a reliable substitute of AASI.

1 Dolan E, Li Y, Thijs L, McCormack P, Staessen JA, O'Brien E, Stanton A. Ambulatory arterial stiffness index: rationale and methodology. Blood Press Monit 2006; 11: 103-105.

2 Li Y, Wang JG, Dolan E, Gao PJ, Guo HF, Nawrot T, Stanton AV, Zhu DL, O'Brien E, Staessen JA. Ambulatory arterial stiffness index derived from 24-h ambulatory blood pressure monitoring. Hypertension 2006; 47: 359-364.

3 Li Y, Dolan E, Wang JG, Thijs L, Zhu DL, Staessen JA, O'Brien E, Stanton A. Ambulatory arterial stiffness index: determinants and outcome. Blood Press Monit 2006: 11: 107-110.

4 Hansen TW, Staessen JA, Torp-Pedersen C, Rasmussen S, Li Y, Dolan E, Thijs L, Wang $J G$, O'Brien E, Ibsen H, Jeppesen J. Ambulatory arterial stiffness index predicts stroke in a general population. J Hypertens 2006; 24: 2247-2253.

5 Dolan E, Thijs L, Li Y, Atkins N, McCormack P, McClory S, O'Brien E, Staessen JA Stanton AV. Ambulatory arterial stiffness index as a predictor of cardiovascular mortality in the Dublin Outcome Study. Hypertension 2006; 47: 365-370.

6 Kikuya M, Staessen JA, Ohkubo T, Thijs L, Metoki H, Asayama K, Obara T, Inoue R, Li Y, Dolan E, Hoshi H, Hashimoto J, Totsune K, Satoh H, Wang JG, O'Brien E, Imai Y.
Ambulatory arterial stiffness index and 24-h ambulatory pulse pressure as predictors of mortality in Ohasama, Japan. Stroke 2007; 38: 1161-1166.

7 Parati G, Stergiou GS, Asmar R, Bilo G, de Leeuw P, Imai Y, Kario K, Lurbe E, Manolis A, Mengden T, O'Brien E, Ohkubo T, Padfield P, Palatini P, Pickering T, Redon J, Revera M, Ruilope LM, Shennan A, Staessen JA, Tisler A, Waeber B, Zanchetti A, Mancia G. European Society of Hypertension guidelines for blood pressure monitoring at home: a summary report of the Second International Consensus Conference on Home Blood Pressure Monitoring. J Hypertens 2008; 26: 1505-1526.

8 Stergiou GS, Argyraki KK, Moyssakis I, Mastorantonakis SE, Achimastos AD, Karamanos VG, Roussias LG. Home blood pressure is as reliable as ambulatory blood pressure in predicting target-organ damage in hypertension. Am J Hypertens 2007; 20: 616-621.

9 Gaborieau V, Delarche N, Gosse P. Ambulatory blood pressure monitoring versus self-measurement of blood pressure at home: correlation with target organ damage. J Hypertens 2008; 26: 1919-1927.

10 Shimbo D, Pickering TG, Spruill TM, Abraham D, Schwartz JE, Gerin W. Relative utility of home, ambulatory, and office blood pressures in the prediction of end-organ damage. Am J Hypertens 2007; 20: 476-482.

11 Stergiou GS, Kalogeropoulos PG, Baibas NM. Prognostic value of home blood pressure measurement. Blood Press Monit 2007; 12: 391-392.

12 Verberk WJ, Kroon AA, Jongen-Vancraybex HA, de Leeuw PW. The applicability of home blood pressure measurement in clinical practice: a review of literature. Vasc Health Risk Manag 2007; 3: 959-966.

13 O'Brien E, Petrie J, Littler W, de Swiet M, Padfield PL, Altman DG, Bland M, Coats A, Atkins N. An outline of the revised British Hypertension Society protocol for the evaluation of blood pressure measuring devices. J Hypertens 1993; 11: 677-679.

14 Gavish B, Ben-Dov IZ, Bursztyn M. Linear relationship between systolic and diastolic blood pressure monitored over $24 \mathrm{~h}$ : assessment and correlates. J Hypertens 2008; 26 : 199-209.

15 Gavish B, Ben-Dov IZ, Kark JD, Mekler J, Bursztyn M. The association of a simple blood pressure-independent parameter derived from ambulatory blood pressure variability with short-term mortality. Hypertens Res 2009; 32: 488-495.

16 Stergiou GS, Kollias A, Rarra VC, Roussias LG. Ambulatory arterial stiffness index: reproducibility of different definitions. Am J Hypertens 2009; 23: 129-134.

17 Qureshi G, Lazar JM, Javaid H, Weber M, Salciccioli L. Self-measured (blood pressure) arterial stiffness index: A promising new measure of arterial stiffness. J Am Soc Hypertens 2007; 1: 347-352.

18 Adiyaman A, Dechering DG, Boggia J, Li Y, Hansen TW, Kikuya M, Bjorklund-Bodegard K, Richart, T Thijs L, Torp-Pedersen C, Ohkubo T, Dolan E, Imai Y, Sandoya E, Ibsen H, Wang J, Lind L, O'Brien E, Thien T, Staessen JA. Determinants of the ambulatory arterial stiffness index in 7604 subjects from 6 populations. Hypertension 2008; 52: 1038-1044.

19 Simonetti GD, von Vigier RO, Wuhl E, Mohaupt MG. Ambulatory arterial stiffness index is increased in hypertensive childhood disease. Pediatr Res 2008; 64: 303-307.

20 Vincenti M, von Vigier RO, Wuhl E, Mohaupt MG, Simonetti GD. The ambulatory arterial stiffness index is not affected by night-time blood pressure characteristics. J Hum Hypertens 2009; 23: 680-682.

21 Schillaci G, Parati G, Pirro M, Pucci G, Mannarino MR, Sperandini L, Mannarino E. Ambulatory arterial stiffness index is not a specific marker of reduced arterial compliance. Hypertension 2007; 49: 986-991.

22 Schillaci G, Parati G. Ambulatory arterial stiffness index: merits and limitations of a simple surrogate measure of arterial compliance. J Hypertens 2008; 26: 182-185.

23 Benetos A, Zureik M, Morcet J, Thomas F, Bean K, Safar M, Ducimetiere P, Guize L. A decrease in diastolic blood pressure combined with an increase in systolic blood pressure is associated with a higher cardiovascular mortality in men. J Am Coll Cardiol 2000; 35: 673-680.

24 Franklin SS, Khan SA, Wong ND, Larson MG, Levy D. Is pulse pressure useful in predicting risk for coronary heart disease? The Framingham heart study. Circulation 1999; 100: 354-360.

25 Verdecchia P, Schillaci G, Borgioni C, Ciucci A, Pede S, Porcellati C. Ambulatory pulse pressure: a potent predictor of total cardiovascular risk in hypertension. Hypertension 1998; 32: 983-988.

26 Jerrard-Dunne P, Mahmud A, Feely J. Ambulatory arterial stiffness index, pulse wave velocity and augmentation index-interchangeable or mutually exclusive measures? J Hypertens 2008; 26: 529-534.

27 Muxfeldt ES, Fiszman R, Castelpoggi CH, Salles GF. Ambulatory arterial stiffness index or pulse pressure: which correlates better with arterial stiffness in resistant hypertension? Hypertens Res 2008; 1: 607-613. 